\title{
Maximum-minimum distance clustering method for split-delivery vehicle-routing problem: Case studies and performance comparisons
}

\author{
Min, J.N. ${ }^{a}$, Jin, C. ${ }^{a,{ }^{*}}$, Lu, L.J. ${ }^{a, b}$ \\ ${ }^{a}$ Taihu University of Wuxi, School of Economics and Management, Jiangsu, P.R. China \\ ${ }^{b}$ Nanjing University, School of Management, Nanjing, Jiangsu, P.R. China
}

\begin{abstract}
A B S T R A C T
The split-delivery vehicle-routing problem in which delivery to a demand point can be served by any number of vehicles is an important branch of classic VRP. Objective function is used to minimise travel distance while using the lowest number of vehicles. According to the maximum-minimum distance clustering method, a three-stage algorithm is proposed. First, the maximumminimum distance method is employed to cluster customer points into the lowest number of groups. Second, according to the maximum vehicle capacity, the load demand in each group is adjusted to create suitable customer points in each clustering group by adopting 'push-out' and 'pull-in' operations. Third, a tabu search is used and an optimised route for each group is generated to minimise the total travel distance. Numerical experiments, some on the benchmark data set, are presented to verify the feasibility and effectiveness of the proposed algorithm. The computational results show that the performance of the proposed algorithm is better in terms of both optimised travel distance and less computation time when the problem size is less than 75. The results also show that when the customer points are in a cluster distribution around the depot, the algorithm achieves better performance.
\end{abstract}

(C) 2019 CPE, University of Maribor. All rights reserved.

\section{ARTICLE INFO}

Keywords:

Split-delivery vehicle-routing problem;

Maximum-minimum distance method;

Load-demand adjustment;

Route optimisation;

Tabu search;

Clustering first and routing later

*Corresponding author:

mjn3862@126.com

(Jin, C.)

Article history:

Received 5 December 2018

Revised 12 February 2019

Accepted 25 February 2019

\section{References}

[1] Dror, M., Trudeau, P. (1989). Savings by split delivery routing, Transportation Science, Vol. 23, No. 2, 141-149, doi: 10.1287/trsc.23.2.141.

[2] Archetti, C., Speranza, M.G. (2012). Vehicle routing problems with split deliveries, International Transactions in Operational Research, Vol. 19, No. 1-2, 3-22, doi: 10.1111/i.1475-3995.2011.00811.x.

[3] Dror, M., Laporte, G., Trudeau, P. (1994). Vehicle routing with split deliveries, Discrete Applied Mathematics, Vol. 50, No. 3, 239-254, doi: 10.1016/0166-218X(92)00172-I.

[4] Archetti, C., Speranza, M.G., Hertz, A. (2006). A tabu search algorithm for the split delivery vehicle routing problem, Transportation Science, Vol. 40, No. 1, 64-73, doi: 10.1287/trsc.1040.0103.

[5] Chen, S., Golden, B., Wasil, E. (2007). The split delivery vehicle routing problem: Applications, algorithms, test problems, and computational results, Networks, Vol. 49, No. 4, 318-329, doi: 10.1002/net.20181.

[6] Archetti, C., Speranza, M.G., Savelsbergh, M.W.P. (2008). An optimization-based heuristic for the split delivery vehicle routing problem, Transportation Science, Vol. 42, No. 1, 22-31, doi: 10.1287/trsc.1070.0204.

[7] Gulczynski, D., Golden, B., Wasil, E. (2010). The split delivery vehicle routing problem with minimum delivery amount, Transportation Research Part E: Logistics and Transportation Review, Vol. 46, No. 5, 612-626, doi: 10.1016/i.tre.2009.12.007.

[8] Wilck IV, J.H., Cavalier, T.M. (2012). A construction heuristic for the split delivery vehicle routing problem, American Journal of Operations Research, Vol. 2, No. 2, 153-162, doi: 10.4236/ajor.2012.22018. 
[9] Liu, W.-S., Yang, F., Li, M.-Q., Chen, P.-Z. (2012). Clustering algorithm for split delivery vehicle routing problem, Control and Decision, Vol. 27, No. 4, 535-541, doi: 10.13195/i.cd.2012.04.57.liuwsh.017.

[10] Lu, X.C., Chen, Q.B., Zhang, Z.J. (2014). The electric vehicle routing optimizing algorithm and the charging stations' layout analysis in Beijing, International Journal of Simulation Modelling, Vol. 13, No. 1, 116-127, doi: 10.2507/IJSIMM13(1)CO4.

[11] Wang, T.-T., Ni, Y.-D., He, W.-L. (2014). Bee colony optimization algorithm for split delivery vehicle routing problem, Journal of Hefei University of Technology, Vol. 37, No. 8, 1015-1019, doi: 10.3969/j.issn.1003-5060.2014.08. $\underline{024}$.

[12] Zhu, X.N., Yan, R., Zhang, Q. (2015). A promoted hybrid heuristic algorithm for two-dimensional multi-depots vehicle routing problem, International Journal of Simulation Modelling, Vol. 14, No. 3, 499-510, doi: 10.2507/ IJSIMM14(3)C011.

[13] Wen, Z.Z. (2015). Researches on iterated local search for the split delivery vehicle routing problem, Beijing Transportation University, Beijing, P.R. China.

[14] Wu, D.Q., Dong, M., Li, H.Y., Li, F. (2016). Vehicle routing problem with time windows using multi-objective coevolutionary approach, International Journal of Simulation Modelling, Vol. 15, No. 4, 742-753, doi: 10.2507/ IJSIMM15(4)CO19.

[15] Tang, M., Gong, D., Liu, S., Zhang, H. (2016). Applying multi-phase particle swarm optimization to solve bulk cargo port scheduling problem, Advances in Production Engineering \& Management, Vol. 11, No. 4, 299-310, doi: 10.14743/apem2016.4.228

[16] Xiang, T., Pan, D. (2016). Clustering algorithm for split delivery vehicle routing problem, Journal of Computer Applications, Vol. 36, No. 11, 3141-3145, doi: 10.11772/i.issn.1001-9081.2016.11.3141.

[17] Cao, Q.K., Yang, K.W., Ren, X.Y. (2017). Vehicle routing optimization with multiple fuzzy time windows based on improved wolf pack algorithm, Advances in Production Engineering \& Management, Vol. 12, No. 4, 401-411, doi: 10.14743/apem017.4.267.

[18] Johanyák, Z.C. (2017). A modified particle swarm optimization algorithm for the optimization of a fuzzy classification subsystem in a series hybrid electric vehicle, Tehnički Vjesnik - Technical Gazette, Vol. 24, Supplement 2, 295-301, doi: 10.17559/TV-20151021202802.

[19] Wang, C.L., Li, S.W. (2018). Hybrid fruit fly optimization algorithm for solving multi-compartment vehicle routing problem in intelligent logistics, Advances in Production Engineering \& Management, Vol. 13, No. 4, 466-478, doi: 10.14743/apem2018.4.304

[20] Belenguer, J.M., Martinez, M.C., Mota, E. (2000). A lower bound for the split delivery vehicle routing problem, Operations Research, Vol. 48, No. 5, 801-810, doi: 10.1287/opre.48.5.801.12407.

[21] Lee, C.-G., Epelman, M.A, White III, C.C., Bozer, Y.A. (2006). A shortest path approach to the multiple-vehicle routing problem with split pick-ups, Transportation Research B: Methodological, Vol. 40, No. 4, 265-284, doi: 10.1016/i.trb.2004.11.004.

[22] Jin, M., Liu, K., Bowden, R.O. (2007). A two-stage algorithm with valid inequalities for the split delivery vehicle routing problem, International Journal of Production Economics, Vol. 105, No. 1, 228-242, doi: 10.1016/i.ijpe. 2006.04.014.

[23] Archetti, C., Bianchessi, N., Speranza, M.G. (2011). A column generation approach for the split delivery vehicle routing problem, Networks, Vol. 58, No. 4, 241-254, doi: 10.1002/net.20467.

[24] Archetti, C., Bianchessi, N., Speranza, M.G. (2015). A branch-price-and-cut algorithm for the commodity constrained split delivery vehicle routing problem, Computers \& Operations Research, Vol. 64, 1-10, doi: 10.1016/ j.cor.2015.04.023.

[25] Luo, Z., Qin, H., Zhu, W., Lim, A. (2017). Branch and price and cut for the split-delivery vehicle routing problem with time windows and linear weight-related cost, Transportation Science, Vol. 51, No. 2, 668-687, doi: 10.1287/ trsc.2015.0666.

[26] Ozbaygin, G., Karasan, O., Yaman, H. (2018). New exact solution approaches for the split delivery vehicle routing problem, EURO Journal on Computational Optimization, Vol. 6, No. 1, 85-115, doi: 10.1007/s13675-017-0089-z.

[27] Min, J., Jin, C., Lu, L. (2018). A three-stage approach for split delivery vehicle routing problem solving, In: Proceedings of 8th International Conference on Logistics, Informatics and Service Sciences (LISS), Toronto, Canada, 16, doi: 10.1109/LISS.2018.8593226.

[28] Zhou, J., Xiong Z.Y., Zhang Y.F., Ren, F. (2006). Multiseed clustering algorithm based on max-min distance algorithm, Journal of Computer Applications, Vol. 26, No. 6, 1425-1427. 


\section{APEM}

\title{
Metoda združevanja največjih in najmanjših razdalj za problem usmerjanja vozil $z$ deljeno dostavo: Študije primerov in primerjave uspešnosti
}

\author{
Min, J.N. ${ }^{a}$, Jin, C. ${ }^{a,}{ }^{*}$, Lu, L.J. ${ }^{a, b}$ \\ ${ }^{\mathrm{a}}$ Taihu University of Wuxi, School of Economics and Management, Jiangsu, P.R. China \\ ${ }^{\mathrm{b}}$ Nanjing University, School of Management, Nanjing, Jiangsu, P.R. China
}

\begin{abstract}
POVZETEK
Problem načrtovanja deljene dostave, pri katerem lahko dostavo do ciljnega mesta opravi poljubno število vozil, je pomembna veja problema usmerjanja vozil (VRP). Ciljna funkcija predvideva zmanjšanje skupne poti ob uporabi najmanjšega števila vozil. V skladu z metodo združevanja po načelu največjanajmanjša razdalja je predlagan tristopenjski algoritem. Najprej se za razvrščanje lokacij odjemalcev $v$ najnižje število skupin uporabi metoda največjanajmanjša razdalja. Nato se, glede na največjo nosilnost vozila prilagodi povpraševanje tako, da se vsaki skupini dodelijo lokacije kupcev z uporabo operacij "push-out" in "pull-in". Nato se uporabi iskanje s tabuji s čimer se ustvari optimizirana pot za vsako skupino in se zmanjša skupna dolžina vseh poti. Za preverjanje izvedljivosti in učinkovitosti predlaganega algoritma so predstavljeni numerični poskusi, nekateri na podlagi referenčnih podatkov. Rezultati kažejo, da je, ko je velikost problema manjša od 75, učinkovitost predlaganega algoritma večja, tako glede na optimizirano razdaljo potovanja kot tudi na krajši čas računanja. Rezultati tudi kažejo, da algoritem doseže boljše delovanje, ko so lokacije kupcev združene okoli skladišča.

(c) 2019 CPE, University of Maribor. All rights reserved.
\end{abstract}

\section{PODATKI O ČLANKU}

Ključne besede:

Problem deljene dostave;

Metoda največja-najmanjša razdalja;

Prilagoditev obremenitve in povpraševanja;

Optimizacija poti;

Iskanje s tabuji;

Združevanje najprej in usmerjanje kasneje

*Kontaktna oseba: mjn3862@126.com (Jin, C.)

Zgodovina članka:

Prejet 5. decembra 2018

Popravljen 12. februarja 2019

Sprejet 25. februarja 2019 